\title{
An Optimal Design of Accelerated Degradation Tests Based on Degradation Performance
}

\author{
Yunshun Wu \\ School of Mathematics and Computer Science, Guizhou Normal University, Guiyang, China \\ Email: wuyunshun1979@163.com
}

How to cite this paper: $\mathrm{Wu}$, Y.S. (2019) An Optimal Design of Accelerated Degradation Tests Based on Degradation Performance. Open Journal of Statistics, 9, 686-694.

https://doi.org/10.4236/ojs.2019.96044

Received: December 2, 2019

Accepted: December 13, 2019

Published: December 16, 2019

Copyright (อ 2019 by author(s) and Scientific Research Publishing Inc. This work is licensed under the Creative Commons Attribution International License (CC BY 4.0).

http://creativecommons.org/licenses/by/4.0/

\begin{abstract}
This paper mainly talks about the relationship between an optimal design of accelerated degradation tests and the degradation performance. When there is a linear relationship between the parameter of distribution and the critical values, a special pattern of accelerated degradation tests is presented by taking the critical values as accelerated variable. And the optimal test plan is obtained by minimizing the asymptotic variance of the MLE of the parameter of distribution. Finally, a numerical example is presented to illustrate the procedures of the test plan.
\end{abstract}

\section{Keywords}

Accelerated Degradation Test, Critical Values, Asymptotic Variance, Optimal Design

\section{Introduction}

With the development of technology, more and more products have higher reliability and longer service life. In many cases, accelerated degradation testing is superior to accelerated life testing in assessing product life. As time goes on, accelerated degradation test has been widely used in reliability evaluation of various products.

There are many factors involved in accelerated degradation test. One of the important aspects is the optimal arrangement or design of the test. In recent years, in the research of experimental optimal design, Boulanger, Escobar [1] and $\mathrm{Yu}$, Tseng [2] gave the cost function of the experiment for the general case. Park and Yum [3] used precision limits for general degradation tests to optimize the tests. Tseng and $\mathrm{Yu}$ [4] proposed a method to optimize the distribution of 
degradation test duration. Yang [5] and $\mathrm{Wu}$, Chang [6] obtained the optimal test design by minimizing the asymptotic variance of quantile life estimation under the restriction of service cost for the constant stress accelerated degradation test. Assuming that the degradation rate obeys the inverse Weibull distribution, $\mathrm{Yu}$ and Tseng [7] designed a constant stress degradation test. Park and Yum [8] designed a step-by-step stress degradation test in which destructive degradation detection was used. Wang, $\mathrm{Xu}$ [9] and Ye, Chen [10] systematically introduced the degradation model of inverse Gaussian process and compared it with gamma process model. Sung and Yum [11] studied the optimal design of accelerated degradation test based on the Wiener process.

In this paper, our main purpose is to find a method based on degradation performance to plan the accelerated degradation tests. We discuss the relationship between the optimal design of a kind of accelerated degradation test and the selection of its degradation of critical value. On condition that there is a linear relationship between the parameter $\mu$ of distribution and the critical values $C$. A special pattern of accelerated degradation tests is presented by taking the critical values as accelerated variable. And the optimal test plan is obtained by minimizing the asymptotic variance of the MLE of the parameter of distribution.

The present paper is structured as follows. In Section 2, we will discuss the relationship between the parameters of failure distribution and the critical value level. In Section 3, we will establish the optimization design model based on degradation performance. Finally, a numerical example is presented to illustrate the procedures of the test plan in Section 4, and Section 5 concludes this paper.

\section{The Critical Values}

For some products with high reliability and long service life, when evaluating their reliability indexes, even if the environmental stress is increased, the failure cannot be or rarely can be observed in the normal time interval. At this time, we use degradation test. Select a performance attribute of the sample, which degrades slowly with time. When its index value exceeds (higher or lower than) a preset limit value, the sample is judged to be invalid. The preset limit is called the degradation threshold. According to reference [12], more failure data can be obtained by selecting the critical value level, and the test time can be shortened.

In this way, a key problem is how to evaluate the failure law of products with the failure data obtained at different critical value levels. This means that the relationship between the parameters of failure distribution and the critical value level should be found. According to literature [13] [14], for some products, the degradation path has a linear form:

$$
D_{i k}(t)=\alpha_{1 k}+\alpha_{2 k} t+\alpha_{3 k} S_{i}+\varepsilon_{i k}(t)
$$

where, $D_{i k}(t)$ is the degradation value of the $k$ th sample at the $i$ th acceleration stress to time $t$.

$\alpha_{1 k}, \alpha_{2 k}, \alpha_{3 k}$ are the coefficient of degradation, $\varepsilon_{i k}(t)$ is the measurement 
error. Now we take the critical value of degradation as $C_{j}$, form $D_{i k}(t)=C_{j}$, We can work out the failure time. Because of $C_{j}=\alpha_{1 k}+\alpha_{2 k} t+\alpha_{3 k} S_{i}+\varepsilon_{i k}(t)$, we obtained $t_{i j k}=\frac{C_{j}-\alpha_{1 k}-\alpha_{3 k} S_{i}}{\alpha_{2 k}}$. This represents the failure time of the $k$ th sample at the $i$ th stress level at the th critical value. Thus, in case of the numbers of invalid samples are $m$, the average life of the sample at the $t$ th critical value and the $i$ th stress level shall be:

$$
\begin{aligned}
\mu_{i j} & =\frac{1}{m} \sum_{k=1}^{m} t_{i j k}=\frac{1}{m} \sum_{k=1}^{m} \frac{C_{j}-\alpha_{1 k}-\alpha_{3 k} S_{i}}{\alpha_{2 k}} \\
& =-\frac{1}{m} \sum_{k=1}^{m} \frac{\alpha_{1 k}}{\alpha_{2 k}}-\frac{1}{m} \sum_{k=1}^{m} \frac{\alpha_{3 k}}{\alpha_{1 k}} S_{i}+\frac{1}{m} \sum_{k=1}^{m} \frac{1}{\alpha_{1 k}} C_{j}
\end{aligned}
$$

Set $\alpha=-\frac{1}{m} \sum_{k=1}^{m} \frac{\alpha_{1 k}}{\alpha_{2 k}}, \quad \beta=-\frac{1}{m} \sum_{k=1}^{m} \frac{\alpha_{3 k}}{\alpha_{1 k}}, \gamma=\frac{1}{m} \sum_{k=1}^{m} \frac{1}{\alpha_{1 k}}$.

Thus, we have $\mu_{i j}=\alpha+\beta \cdot S_{i}+\gamma \cdot C_{j}$.

Therefore, the average life is related to stress and critical value as follows:

$$
\mu=\alpha+\beta \cdot S+\gamma \cdot C
$$

In this way, as mentioned above, more failure data can be obtained by selecting the critical value level, and the test time can be shortened, so as to accelerate the test.

\section{A special Optimization Design}

\subsection{Test Description and Assumption}

\section{Test description}

The number of test samples is $n$. All samples are subjected to constant stress accelerated degradation test at $q$ different but constant stress levels. The acceleration stress level (or its deformation) is $S_{1}, S_{2}, \cdots, S_{q} \quad\left(S_{0}<S_{1}<S_{2}<\cdots<S_{q}\right.$, $S_{0}$ is the normal stress level, $S_{q}$ is the highest stress level). The ratio of samples is $\pi_{i}$ to the stress level $S_{i}$. So the Sample number is $n_{i}=n \cdot \pi_{i}$ at the stress level $S_{i}$. The test adopts timing truncation. The closing time is $\tau_{i}$. $D$ represents the degradation amount of sample performance characteristics. We take $m$ critical values for the degradation. Respectively $C_{1}, C_{2}, \cdots, C_{m}$, where $C_{m}$ is an extreme threshold, $C_{0}$ represents the critical value of degradation under normal stress.

For discussion purposes, we first standardize the stress levels as follows: Set $X_{i}=\frac{S_{i}-S_{q}}{S_{0}-S_{q}}, i=0,1,2, \cdots, q$, thus, we obtain $1=X_{0}>X_{1}>\cdots>X_{q}=0$. The advantage is that the range of stress change is limited to the interval $[0,1]$. The level of the threshold is then standardized as follows:

$Y_{j}=\frac{C_{j}-C_{m}}{C_{0}-C_{m}}, j=1,2, \cdots, m$, then $Y_{0}=1, Y_{m}=0$. And all $Y_{j}$ are between 0 and 1. 


\section{Test assumption}

1) Failure time follows lognormal distribution $\operatorname{LN}\left(\mu, \sigma^{2}\right)$;

2) Parameter $\mu$ is a linear function of test stress and degradation critical value (from Equation (1)), hence:

$$
\mu=\mu\left(X_{i}, Y_{j}\right)=\alpha+\beta X_{i}+\gamma Y_{j}
$$

3) The parameters $\sigma$ do not change with the change of test stress and degradation critical value.

\subsection{Optimization Design Model}

In the above case, we hope to arrange the experiment based on some standards, so that the experiment can be optimized to a certain extent. Here, the experiment is arranged by minimizing the estimated asymptotic variance $A \operatorname{Var}(\hat{\mu}(1,1))$ of the parameters $\mu$ in the normal environment (the stress level is taken $X_{0}=1$, while the degradation critical value is taken $Y_{0}=1$ ).

Maximum likelihood estimation of model parameters:

According to the experimental description and assumption, at a sample observation value, its log likelihood function is

$$
L_{i, j, k}=I_{i, j, k}\left(-\frac{1}{2} \ln (2 \pi)-\ln \sigma-\frac{1}{2} z_{i, j, k}^{2}\right)+\left(1-I_{i, j, k}\right) \ln \left(1-\Phi\left(\eta_{i, j}\right)\right)
$$

where $z_{i, j, k}=t_{i, j, k}-\alpha-\beta X_{i}-\gamma Y_{j}, \quad \eta_{i, j}=\tau_{i}-\alpha-\beta X_{i}-\gamma Y_{j}$.

When $t_{i, j, k} \leq \tau_{i}, I_{i, j, k}=1$; otherwise $I_{i, j, k}=0 . \Phi(\cdot)$ is the distribution function of standard normal distribution. According to the description of the test, each sample has $m$ degradation critical value to judge whether it is invalid. Therefore, $m \cdot n$ observations will be obtained for $n$ samples. From their independence, the log likelihood function of all observations is:

$$
L=\sum_{i=1}^{q} \sum_{j=1}^{m} \sum_{k=1}^{n_{i}} L_{i, j, k}
$$

The maximum likelihood estimation $\hat{\alpha}, \hat{\beta}, \hat{\gamma}, \hat{\sigma}$ of parameters $\alpha, \beta, \gamma, \sigma$ can be obtained by maximizing $L$. At this time, the analytical expression cannot be obtained. The numerical results can be obtained by using the nonlinear programming method in reference [15].

Taking $i=j=0$ in the Equation (2), and let $X_{0}=1, Y_{0}=1$, we obtain

$$
\mu(1,1)=\mu\left(X_{0}, Y_{0}\right)=\alpha+\beta X_{0}+\gamma Y_{0}=\alpha+\beta+\gamma
$$

Thus, the estimate of $\mu$ is

$$
\hat{\mu}(1,1)=\hat{\alpha}+\hat{\beta}+\hat{\gamma}
$$

In order to obtain the progressive variance of $\hat{\mu}(1,1)$, we first find the Fisher information matrix of parameter maximum likelihood estimation.

According to the above form of log likelihood function $L_{i, j, k}$, We can get the Fisher information matrix of an observation value as follows 


$$
\begin{aligned}
& {\left[E\left(-\frac{\partial^{2} L_{i, j, k}}{\partial^{2} \alpha}\right) E\left(-\frac{\partial^{2} L_{i, j, k}}{\partial \alpha \partial \beta}\right) E\left(-\frac{\partial^{2} L_{i, j, k}}{\partial \alpha \partial \gamma}\right) E\left(-\frac{\partial^{2} L_{i, j, k}}{\partial \alpha \partial \sigma}\right)\right]} \\
& F_{i, j}=\left|\begin{array}{llll}
E\left(-\frac{\partial^{2} L_{i, j, k}}{\partial \alpha \partial \beta}\right) & E\left(-\frac{\partial^{2} L_{i, j, k}}{\partial^{2} \beta}\right) & E\left(-\frac{\partial^{2} L_{i, j, k}}{\partial \beta \partial \gamma}\right) & E\left(-\frac{\partial^{2} L_{i, j, k}}{\partial \beta \partial \sigma}\right) \\
E\left(-\frac{\partial^{2} L_{i, j, k}}{\partial \alpha \partial \gamma}\right) & E\left(-\frac{\partial^{2} L_{i, j, k}}{\partial \beta \partial \gamma}\right) & E\left(-\frac{\partial^{2} L_{i, j, k}}{\partial^{2} \gamma}\right) & E\left(-\frac{\partial^{2} L_{i, j, k}}{\partial \gamma \partial \sigma}\right)
\end{array}\right| \\
& \left.E\left(-\frac{\partial^{2} L_{i, j, k}}{\partial \alpha \partial \sigma}\right) E\left(-\frac{\partial^{2} L_{i, j, k}}{\partial \beta \partial \sigma}\right) E\left(-\frac{\partial^{2} L_{i, j, k}}{\partial \gamma \partial \sigma}\right) E\left(-\frac{\partial^{2} L_{i, j, k}}{\partial^{2} \sigma}\right)\right] \\
& =\frac{1}{\sigma^{2}}\left[\begin{array}{cccc}
A_{i, j} & X_{i} A_{i, j} & Y_{j} A_{i, j} & B_{i, j} \\
X_{i} A_{i, j} & X_{i}^{2} A_{i, j} & X_{i} Y_{j} A_{i, j} & X_{i} B_{i, j} \\
Y_{j} A_{i, j} & X_{i} Y_{j} A_{i, j} & Y_{j}^{2} A_{i, j} & Y_{j} B_{i, j} \\
B_{i, j} & X_{i} B_{i, j} & Y_{j} B_{i, j} & C_{i, j}
\end{array}\right]
\end{aligned}
$$

where,

$$
\begin{gathered}
A_{i, j}=\Phi\left(\eta_{i, j}\right)-\phi\left(\eta_{i, j}\right)\left(\eta_{i, j}-\frac{\phi\left(\eta_{i, j}\right)}{1-\Phi\left(\eta_{i, j}\right)}\right), \\
B_{i, j}=-\phi\left(\eta_{i, j}\right)\left\{1+\eta_{i, j}\left(\eta_{i, j}-\frac{\phi\left(\eta_{i, j}\right)}{1-\Phi\left(\eta_{i, j}\right)}\right)\right\} \\
C_{i, j}=2 \Phi\left(\eta_{i, j}\right)-\eta_{i, j} \phi\left(\eta_{i, j}\right)\left(1+\eta_{i, j}^{2}-\frac{\eta_{i, j} \phi\left(\eta_{i, j}\right)}{1-\Phi\left(\eta_{i, j}\right)}\right),
\end{gathered}
$$

$\phi(\cdot)$ is the distribution function of standard normal distribution.

Thus, the Fisher information matrix of all observations is $F=\sum_{i=}^{q} \sum_{j=1}^{m} n \pi_{i} F_{i, j}$.

So, the covariance matrix of the MLE $\hat{\alpha}, \hat{\beta}, \hat{\gamma}, \hat{\sigma}$ of the parameter $\alpha, \beta, \gamma, \sigma$ can be calculated as

$$
\Sigma=\left[\begin{array}{cccc}
\operatorname{Var}(\hat{\alpha}) & \operatorname{Cov}(\hat{\alpha}, \hat{\beta}) & \operatorname{Cov}(\hat{\alpha}, \hat{\gamma}) & \operatorname{Cov}(\hat{\alpha}, \hat{\sigma}) \\
\operatorname{Cov}(\hat{\alpha}, \hat{\beta}) & \operatorname{Var}(\hat{\beta}) & \operatorname{Cov}(\hat{\beta}, \hat{\gamma}) & \operatorname{Cov}(\hat{\beta}, \hat{\sigma}) \\
\operatorname{Cov}(\hat{\alpha}, \hat{\gamma}) & \operatorname{Cov}(\hat{\beta}, \hat{\gamma}) & \operatorname{Var}(\hat{\gamma}) & \operatorname{Cov}(\hat{\gamma}, \sigma) \\
\operatorname{Cov}(\hat{\alpha}, \hat{\sigma}) & \operatorname{Cov}(\hat{\beta}, \hat{\sigma}) & \operatorname{Cov}(\hat{\gamma}, \hat{\sigma}) & \operatorname{Var}(\hat{\sigma})
\end{array}\right]=F^{-1}
$$

According to the theory of maximum likelihood estimation and (3), we have

$$
h^{\mathrm{T}}=\left(\frac{\partial \hat{\mu}(1,1)}{\partial \alpha}, \frac{\partial \hat{\mu}(1,1)}{\partial \beta}, \frac{\partial \hat{\mu}(1,1)}{\partial \gamma}, \frac{\partial \hat{\mu}(1,1)}{\partial \sigma}\right)=(1,1,1,0)
$$

Thus, we obtain

$$
A \operatorname{Var}(\hat{\mu}(1,1))=h^{\mathrm{T}} \cdot \Sigma \cdot h=(1,1,1,0) \cdot \Sigma \cdot(1,1,1,0)^{\mathrm{T}}
$$

In general, before the test, the maximum stress that the sample can withstand and the extreme critical value of degradation have been determined. Therefore, 
when the sample size $n$ is determined, we can select the value of $X_{i}, Y_{j}, \pi_{i}$ to minimize the above-mentioned progressive variance

$A \operatorname{Var}(\hat{\mu}(1,1))=(1,1,1,0) \cdot \Sigma \cdot(1,1,1,0)^{\mathrm{T}}$. So the optimal model can be described as follows

$$
\operatorname{Min}: \operatorname{AVar}(\hat{\mu}(1,1))=(1,1,1,0) \cdot \Sigma \cdot(1,1,1,0)^{\mathrm{T}}
$$

where, the decision variables are: $X_{i}, Y_{j}, \pi_{i}, i=1,2, \cdots, q-1$; $j=1,2, \cdots, m-1$, and $0 \leq X_{i}, Y_{j}, \pi_{i} \leq 1$.

\subsection{A Special Case of Optimization Model}

As a special case of optimization model, we set $q=2, m=2$ to calculate $A \operatorname{Var}(\hat{\mu}(1,1))$.

In this case, the Fisher information matrix is $F=\sum_{i=}^{2} \sum_{j=1}^{2} n \pi_{i} F_{i, j}=\frac{n}{\sigma^{2}} F_{0}$, $F_{0}=\left(f_{i, j}\right)_{4 \times 4}$ is a fourth-order matrix, where:

$$
\begin{gathered}
f_{1,1}=\sum_{i=1}^{2} \sum_{j=1}^{2} \pi_{i} A_{i, j}, \quad f_{2,2}=\sum_{i=1}^{2} \sum_{j=1}^{2} X_{i}^{2} \pi_{i} A_{i, j}, \quad f_{3,3}=\sum_{i=1}^{2} \sum_{j=1}^{2} Y_{j}^{2} \pi_{i} A_{i, j}, \\
f_{4,4}=\sum_{i=1}^{2} \sum_{j=1}^{2} \pi_{i} C_{i, j}, \quad f_{1,2}=f_{2,1}=\sum_{i=1}^{2} \sum_{j=1}^{2} X_{i} \pi_{i} A_{i, j}, \quad f_{1,3}=f_{3,1}=\sum_{i=1}^{2} \sum_{j=1}^{2} Y_{j} \pi_{i} A_{i, j}, \\
f_{1,4}=f_{4,1}=\sum_{i=1}^{2} \sum_{j=1}^{2} \pi_{i} B_{i, j}, \quad f_{2,3}=f_{3,2}=\sum_{i=1}^{2} \sum_{j=1}^{2} X_{i} Y_{j} \pi_{i} A_{i, j}, \\
f_{2,4}=f_{4,2}=\sum_{i=1}^{2} \sum_{j=1}^{2} X_{i} \pi_{i} B_{i, j}, \quad f_{3,4}=f_{4,3}=\sum_{i=1}^{2} \sum_{j=1}^{2} Y_{j} \pi_{i} B_{i, j},
\end{gathered}
$$

Substituting the above results into (5), we obtain

$$
A \operatorname{Var}(\hat{\mu}(1,1))=(1,1,1,0) \cdot \Sigma \cdot(1,1,1,0)^{\mathrm{T}}=(1,1,1,0) \cdot F_{0}^{-1} \cdot(1,1,1,0)^{\mathrm{T}}=\frac{n}{\sigma^{2}} V
$$

where, $V=\frac{E_{1}+E_{2}+E_{3}+2\left(E_{4}+E_{5}+E_{6}\right)}{\left|F_{0}\right|},\left|F_{0}\right|$ is the determinant of matrix $F_{0}$.

$$
\begin{gathered}
E_{1}=f_{2,2} f_{3,3} f_{4,4}+2 f_{2,3} f_{3,4} f_{4,2}-f_{2,4}^{2} f_{3,3}-f_{3,4}^{2} f_{2,2}-f_{2,3}^{2} f_{4,4} \\
E_{2}=f_{1,4} f_{2,4} f_{3,3}+f_{1,2} f_{3,4}^{2}+f_{1,3} f_{2,3}^{2} f_{4,4}-f_{1,2} f_{3,3} f_{4,4}-f_{1,4} f_{2,3} f_{3,4}-f_{1,3} f_{2,4} f_{3,4} \\
E_{3}=f_{1,2} f_{2,3} f_{4,4}+f_{1,4} f_{2,2} f_{3,4}+f_{1,3} f_{2,4}^{2}-f_{1,4} f_{2,3} f_{2,4}-f_{1,2} f_{2,4} f_{3,4}-f_{1,3} f_{2,2} f_{4,4} \\
E_{4}=f_{1,1} f_{3,3} f_{4,4}+2 f_{1,3} f_{1,4} f_{3,4}-f_{1,4}^{2} f_{3,3}-f_{1,1} f_{3,4}^{2}-f_{1,3}^{2} f_{4,4} \\
E_{5}=f_{1,4}^{2} f_{2,3}+f_{1,1} f_{2,4} f_{3,4}+f_{1,2} f_{1,3} f_{4,4}-f_{1,1} f_{2,3} f_{4,4}-f_{1,2} f_{1,4} f_{3,4}-f_{1,4} f_{1,3} f_{2,4} \\
E_{6}=f_{1,1} f_{2,2} f_{4,4}+2 f_{1,2} f_{1,4} f_{2,4}-f_{1,4}^{2} f_{2,2}-f_{1,1} f_{2,4}^{2}-f_{1,2}^{2} f_{4,4}
\end{gathered}
$$

Because the total number $n$ of samples and $\sigma^{2}$ are constant, the optimization model is:

$$
\operatorname{Min}: V=\frac{E_{1}+E_{2}+E_{3}+2\left(E_{4}+E_{5}+E_{6}\right)}{\left|F_{0}\right|}
$$


where, the decision variables are: $X_{1}, Y_{1}, \pi_{1}$, and $0 \leq X_{1}, Y_{1}, \pi_{1} \leq 1$.

This nonlinear programming problem can be solved by the method of reference [15].

\section{Numerical Example}

In order to illustrate the above optimization process, we take 50 LEDs for accelerated degradation test. The degraded performance attribute is taken as its luminous intensity. The acceleration variable of the test is taken as voltage. Under normal working conditions, the working current of the sample is $40 \mathrm{~mA}$. According to previous experience, the maximum allowable working current is 330 $\mathrm{mA}$ (without changing its failure mechanism). Here we consider accelerated degradation tests at two stress levels. The voltage level is $S_{1}, S_{2} .40<S_{1}<330$, $S_{2}=330$. When the sample is working under normal conditions, if its luminous intensity is lower than $50 \%$ of its initial intensity, it will be judged as invalid. If the initial luminous intensity is set to 1 , the critical value under normal conditions is $C_{0}=0.5$. Now, we accelerate the degradation threshold at the same time. Set the extreme critical value as $C_{2}=0.1$. We optimize the experimental arrangement according to the above model.

The estimation of parameters in the model can be based on previous experience or similar data. Here, we set its estimate as $\hat{\alpha}=5.5, \hat{\beta}=6, \hat{\gamma}=3$, $\hat{\sigma}=1$. We take the cut-off time of the test at two stress levels as $\tau_{1}=\tau_{2}=8$. Put the above data into the optimization model (6), using the nonlinear programming method, we obtain $X_{1}=0.412, Y_{1}=0.646, \pi_{1}=0.557$

Thus, we have $n_{1}=n \cdot \pi_{1}=50 \times 0.557 \approx 28, n_{2}=n-n_{1}=22$.

And $X_{1}=\frac{S_{1}-S_{2}}{S_{0}-S_{2}}, S_{1}=210.5$; and $Y_{1}=\frac{C_{1}-C_{2}}{C_{0}-C_{2}}, C_{1}=0.24$.

Therefore, the optimal design of the test is: Take 28 samples and put them into $210.5 \mathrm{~mA}$ for accelerated degradation test. When the luminous intensity of the test sample-led is lower than $24 \%$ of the initial value, we determine that the sample is invalid once. When the luminous intensity of the test sample is lower than $50 \%$ of the initial value, we decide that it will fail again. The closing time of the test is $t=8$. In addition, 22 samples are placed at $330 \mathrm{~mA}$ for accelerated degradation test. When the luminous intensity of the test sample is lower than $24 \%$ of the initial value, we determine that the sample is invalid once. When the luminous intensity of the test sample is lower than $50 \%$ of the initial value, we decide that it will fail again. The closing time of the test is $t=8$.

\section{Results and Conclusion}

In this work we established an optimization design model based on degradation performance for accelerated degradation test. The model shows that the test can be accelerated by selecting the critical value level. For ease of application, we simplified the model, and presented a numerical example to illustrate the procedures of the test plan. 


\section{Acknowledgements}

This research was partially supported by the $\mathrm{PhD}$ research startup foundation of Guizhou Normal University (Grant No. GZNUD[2017]27) \& Science and Technology Foundation of Guizhou Province (LKS[2012]11), China. \& Teaching Project of Guizhou Normal University in 2016: Contract No. [2016] XJ No. 09.

\section{Conflicts of Interest}

The author declares no conflicts of interest regarding the publication of this paper.

\section{References}

[1] Boulanger, M. and Escobar, L.A. (1994) Experimental Design for a Class of Accelerated Degradation Tests. Technometrics, 36, 260-272. https://doi.org/10.1080/00401706.1994.10485803

[2] Yu, H.F. and Tseng, S.T. (1999) Designing a Degradation Experiment. Naval Research Logistics, 46, 689-706. https://doi.org/10.1002/(SICI)1520-6750(199909)46:6<689::AID-NAV6>3.0.CO;2-N

[3] Park, J.I. and Yum, B.J. (1997) Optimal Design of Accelerated Degradation Tests for Estimating Mean Lifetime at the Use Condition. Engineering Optimization, 28, 199-230. https://doi.org/10.1080/03052159708941132

[4] Tseng, S.T. and Yu, H.F. (1997) A Termination Rule for Degradation Experiments. IEEE Transactions on Reliability, 46, 130-133. https://doi.org/10.1109/24.589938

[5] Yang, G. and Yang, K. (2002) Accelerated Degradation-Tests with Tightened Critical Values. IEEE Transactions on Reliability, 51, 463-468. https://doi.org/10.1109/TR.2002.804490

[6] Wu, S.J. and Chang, C.T. (2002) Optimal Design of Degradation Tests in Presence of Cost Constraint. Reliability Engineering \& System Safety, 76, 109-115. https://doi.org/10.1016/S0951-8320(01)00123-5

[7] Yu, H.F. and Tseng, S.T. (2004) Designing a Degradation Experiment with a Reciprocal Weibull Degradation Rate. Quality Technology \& Quantitative Management, 1, 47-63. https://doi.org/10.1080/16843703.2004.11673064

[8] Park, S.J., Yum, B. J. and Balamurali, S. (2004) Optimal Design of Step-Stress Degradation Tests in the Case of Destructive Measurement. Quality Technology \& Quantitative Management, 1, 105-124. https://doi.org/10.1080/16843703.2004.11673067

[9] Wang, X. and Xu, D. (2010) An Inverse Gaussian Process Model for Degradation Data. Technometrics, 52, 188-197. https://doi.org/10.1198/TECH.2009.08197

[10] Ye, Z.S. and Chen, N. (2014) The Inverse Gaussian Process as a Degradation Model. Technometrics, 56, 302-311. https://doi.org/10.1080/00401706.2013.830074

[11] Sung, S.I. and Yum, B.J. (2016) Optimal Design of Step-Stress Accelerated Degradation Tests Based on the Wiener Degradation Process. Quality Technology \& Quantitative Management, 13, 367-393. https://doi.org/10.1080/16843703.2016.1189179

[12] Yang, K. and Yang, G. (1998) Degradation Reliability Assessment Using Severe Critical Values. International Journal of Reliability, Quality and Safety Engineering, 5, 85-95. https://doi.org/10.1142/S0218539398000091

[13] Lu, J.C. and Yang, P.Q. (1997) Statistical Inference of a Time-to-Failure Distribu- 
tion Derived from Linear Degradation Data. Technometrics, 39, 391-400. https://doi.org/10.1080/00401706.1997.10485158

[14] Lall, P., Pecht, M. and Hakim, E. (1997) Influence of Temperature on Microelectronics and System Reliability. CRC Press, London.

[15] Bazaraa, M. and Shetty, C. (1979) Nonlinear Programming: Theory and Algorithms. John Wiley \& Sons, New York. 